\title{
Loss of stability and hydrophobicity of presenilin 1 mutations causing Alzheimer's Disease
}

\section{Somavarapu, Arun Kumar; Kepp, Kasper Planeta}

Published in:

Journal of Neurochemistry

Link to article, DOI:

10.1111/jnc. 13535

Publication date:

2016

Document Version

Publisher's PDF, also known as Version of record

Link back to DTU Orbit

Citation $(A P A)$ :

Somavarapu, A. K., \& Kepp, K. P. (2016). Loss of stability and hydrophobicity of presenilin 1 mutations causing Alzheimer's Disease. Journal of Neurochemistry, 137(1), 101-111. https://doi.org/10.1111/jnc.13535

\section{General rights}

Copyright and moral rights for the publications made accessible in the public portal are retained by the authors and/or other copyright owners and it is a condition of accessing publications that users recognise and abide by the legal requirements associated with these rights.

- Users may download and print one copy of any publication from the public portal for the purpose of private study or research.

- You may not further distribute the material or use it for any profit-making activity or commercial gain

- You may freely distribute the URL identifying the publication in the public portal 


\title{
ORIGINAL Loss of stability and hydrophobicity of presenilin 1 ARTICLE mutations causing Alzheimer's disease
}

\author{
Arun Kumar Somavarapu and Kasper P. Kepp \\ Technical University of Denmark, DTU Chemistry, Kongens Lyngby, Denmark
}

\begin{abstract}
Nearly 200 mutations in the gene coding for presenilin 1 (PSEN1) cause early-onset Alzheimer's disease, yet the molecular mechanism remains obscure. As a meta-analysis, we compiled available clinical and biochemical data for PSEN1 variants and correlated these to chemical properties of the mutants. We found statistically significant relationships between relative $A \beta_{42}$ levels and clinical age of onset. We then computed chemical properties of the mutants from a variety of computational chemistry tools. Relative $A \beta_{42}$ levels correlated significantly $(95 \%$ confidence or more from $p$-values of linear regression) with loss of hydrophobicity for four different regression analyses (squared correlation coefficient of linear regression $R^{2}$ of $\left.0.41-0.53\right)$ and with increased polarity $\left(R^{2}=0.47\right.$, $0.59)$ and loss of protein stability $\left(R^{2}=0.39,0.63\right)$ for two
\end{abstract}

independent data sets. Age of onset of patients carrying PSEN1 variants correlated with increased polarity $\left(R^{2}=0.49,0.40\right)$ and loss of stability $\left(R^{2}=0.75,0.44\right)$ of the protein for both data sets. These relations suggest that mutants impair the membrane-associated structural integrity of presenilin by reducing hydrophobic membrane association and overall protein stability. This explains why the many mutations that spread out across the protein and far from the catalytic aspartates can cause disease. The identified molecular determinants of clinical age of symptom onset may be relevant to future presenilinmodulating therapies specifically directed towards increasing the structural integrity and packing of the protein.

Keywords: Alzheimer's Disease, chemical properties, genotype-phenotype relation, mutation, Presenilin.

J. Neurochem. (2016) 137, 101-111.
Alzheimer's Disease (AD) is a devastating neurological disease that affects millions of people worldwide, with growing prevalence but no efficient cure (Goedert and Spillantini 2006; Karran et al. 2011; Kepp 2012). Among the characteristic biochemical features of the disease, tau-protein hyperphosphorylation (Goedert and Spillantini 2006), oxidative stress (Jomova et al. 2010), metal ion dysregulation (Kepp 2012) (Bush 2013), metabolic dysfunctions (Morris et al. 2014; Wilkins et al. 2014) and extracellular deposition of fibrils ('senile plaques') consisting of amyloid- $\beta$ (A $\beta$ ) peptides (Hardy and Selkoe 2002; Karran et al. 2011) consistently occur.

AD mainly occurs sporadically, with little family history. However, genetic mutations in the genes coding for amyloid precursor protein (APP) (Goate et al. 1991), presenilin 1 (PSEN1) (Sherrington et al. 1995) and presenilin 2 (PSEN2) (Levy-Lahad et al. 1995; Rogaev et al. 1995) cause specific forms of early-onset familial AD (FAD) (Campion et al. 1999; Hollingworth et al. 2011; Ryman et al. 2014). The A $\beta$ peptides are considered central to AD (the 'amyloid hypothesis', see Hardy and Selkoe 2002), because of the A $\beta$ deposits in patient brains and the fact that APP and presenilin relate directly to $A \beta$ (Hardy and Selkoe 2002; Tiwari and Kepp 2015); A $\beta$ is cleaved from membrane-bound APP by the action of several enzymes, the final one being $\gamma$-secretase, a protein complex whose catalytic component is presenilin (De Strooper et al. 1998; Kimberly et al. 2003; ChávezGutiérrez et al. 2012).

Despite massive research, current treatments still only delay $\mathrm{AD}$ by some months. Moreover, several recent highprofile clinical trials of new AD medicine have failed, which has lead to the criticism of current paradigms and protocols

Received November 12, 2015; revised manuscript received December 23, 2015; accepted December 29, 2015.

Address correspondence and reprint requests to Kasper P. Kepp, Technical University of Denmark, DTU Chemistry, DK-2800 Kongens Lyngby, Denmark. E-mail: kpj@kemi.dtu.dk

Abbreviations used: AD, Alzheimer's disease; APP, amyloid precursor protein; $A \beta_{40}$ and $A \beta_{42}$, amyloid- $\beta$, isoform with 40 and 42 residues, respectively; $\mathrm{A} \beta$, amyloid- $\beta$; FAD, familial early-onset Alzheimer's disease; K\&D, hydrophobicity scale of Kyte and Doolittle; PSEN1, the gene coding for the protein Presenilin 1; PSEN2, the gene coding for Presenilin $2 ; R^{2}$, squared correlation coefficient from linear regression analysis. 
and lack of molecular insight (Teich and Arancio 2012; Doody et al. 2013; De Strooper 2014; Karran and Hardy 2014; Rosenblum 2014). Accordingly, etiologies remain under debate with multiple hypotheses in existence (Carvalho et al. 2009; DeToma et al. 2012; Kepp 2012; Teich and Arancio 2012; Morris et al. 2014). Specifically, two hypotheses can explain PSEN-related FAD: Gain of toxic amyloid function or loss of normal presenilin function (Shen and Kelleher 2007).

The A $\beta$ peptides produced by $\gamma$-secretase from APP vary in length (Haass and Selkoe 1993; Haass et al. 2012). Among the produced isoforms, $A \beta_{40}$ and $A \beta_{42}$ dominate, and the longer isoforms tend to be more hydrophobic, more aggregation-prone and also more toxic in cell assays (Rauk 2009). Accordingly, higher ratios of long (e.g. $A \beta_{42}$ ) versus short amyloids (e.g. $A \beta_{40}$ ) are suggested to be pathogenic culprits of AD (Karran et al. 2011; Wolfe 2012). Most genetic variants of PSEN1 and APP increase this ratio (Borchelt et al. 1996; Duff et al. 1996; Lemere et al. 1996), consistent with a toxic gain-of-function mechanism. Increased long/short $\mathrm{A} \beta$ ratios may be caused by delays in the sequential proteolysis by mutant $\gamma$-secretase (Wolfe 2012; Fernandez et al. 2014). Soluble oligomers of $A \beta_{42}$ are particularly toxic and may be pathogenic forms of $A \beta$ (Hardy and Selkoe 2002; Karran et al. 2011; Hefti et al. 2013).

However, loss of normal presenilin function could also cause FAD (De Strooper 2007; Shen and Kelleher 2007): The catalytic proficiency and total $\mathrm{A} \beta$ levels produced by PSEN1 mutants tend to decrease (Cacquevel et al. 2012); $\gamma$ secretase cleaves other substrates including the important Notch protein, and PSEN1 knock-out models produce neurodegeneration (Saura et al. 2004), although not all FAD-causing PSEN1 mutations lose their 'non'- $\gamma$-secretase functions (Woodruff et al. 2013). Among possible functions of the presenilin protein, a role in calcium homeostasis is notable as it is crucial for neuronal homeostasis and signaling (Supnet and Bezprozvanny 2011); (Corona et al. 2011). In this context, $\mathrm{A} \beta$ also forms calcium channels (Kuo et al. 2015), providing an alternative presenilin/APP unifying lossof-function etiology (Arispe et al. 1993; Small et al. 2009; Demuro et al. 2010).

The 3-dimensional structure of human $\gamma$-secretase was recently determined at $4.5,4.3$ and $3.4 \AA$ using singleparticle cryoelectron microscopy (Lu et al. 2014; Bai et al. 2015; Sun et al. 2015) to reveal a horse-shoe like transmembrane structure with presenilin having nine transmembrane helices. This insight into the second and tertiary structure of presenilin serves as a unique opportunity to study the chemical forces of PSEN1 mutations that lead to FAD within a structural and chemical property context.

As shown recently, age of symptom onset differs substantially and significantly among the different PSEN1 variants, rendering this descriptor useful for investigating the disease (Ryman et al. 2014). In this work, we compiled both the available clinical data for age of symptom onset of PSEN1 variant carriers and the available data for $A \beta_{42} / A \beta_{40}$ levels upon expression of PSEN1 mutants, as a meta-analysis extending previous attempts to identify such correlations (Duering et al. 2005). As a new approach, we correlated these clinical data against computed chemical and structural properties of the corresponding PSEN1 mutants. We found statistically significant correlations between age of onset and relative $\mathrm{A} \beta_{42}$ levels, as previously found (Duering et al. 2005), but more interestingly we also found significant correlations with specific changes in fundamental chemical properties of the mutants, associated with increased polarity and reduced protein stability. These correlations to fundamental chemical properties are the first reported, as far as we are aware.

The findings can be rationalized by a model where the different regions of presenilin need to retain hydrophobic packing and stability to adhere well to the membrane-bound protein, whereas increased polarity or loss of stability will reduce the packing of presenilin within the membrane and the remaining enzyme complex. This general stabilityassociation mechanism provides the first chemical-propertyderived explanation as to why so many mutations are scattered throughout the sequence of PSEN1, usually far from the catalytic aspartates, and how all of this can lead to disease. Experiments have demonstrated that the hydrophobic bilayer thickness is critical for both total catalytic activity as well as cleavage specificity, as measured by $A \beta$ isoform ratios, of $\gamma$-secretase, with production of $\mathrm{A} \beta_{40}$ being higher in thicker membranes (Winkler et al. 2012). These experiments are consistent with a membrane-packing integrity model as deduced from the chemical properties correlating with age of symptom onset in this work.

\section{Methods}

Data collection

PSEN1 mutations linked to autosomal dominant AD were compiled from the Alzforum database (www.alzforum.org) and the AD \& FTD database (www.molgen.ua.ac.be/ADMutations). The data set contains a total of 210 mutations of which 183 are pathogenic point missense mutations, 12 are non-pathogenic point missense mutations, and 15 correspond to insertions, deletions and frameshifts. The subset containing pathogenic missense mutations were used for further analysis. Among 183 such mutations, multiple mutations occurred at 53 positions, which together constitute nearly two-thirds of all pathogenic mutations. The mutants are collected in Table S1.

For as many PSEN1 mutants as possible, measured $A \beta_{42} / A \beta_{40}$ levels, relative $A \beta_{42} / A \beta_{\text {total }}$ ratios and the mean clinical age of symptom onset were collected from the databases and literature. Because of differences in protocols, the data set in some instances contains different quantifications of relative $A \beta_{42}$ levels: $A \beta_{42} / A \beta_{40}$ denotes the ratio of total $A \beta_{42}$ to the total amount of the $A \beta_{40}$ isoform as expressed in cell models of the mutant, whereas $A \beta_{42} /$ $\mathrm{A} \beta_{\text {total }}$ denotes the ratio of $\mathrm{A} \beta_{42}$ to the total $\mathrm{A} \beta$ (all isoforms, almost 
exclusively $A \beta_{42}$ and $A \beta_{40}$ ); both ratios were reported as ratios relative to the ratio expressed by wild type presenilin in the same experiment. As a result of these differences, we divided the total data set into two individual data sets (data set 1 and 2).

\section{Computation of chemical properties}

To have a molecular structure of presenilin relevant for computing structure-dependent properties, we used a presenilin homology model that was modeled using the Modeller program (Sali and Blundell 1993) and the SWISSMODEL homology server (Arnold et al. 2006; Biasini et al. 2014). This homology model preserves the transmembrane helix topology of the recent electron microscopy structure at $3.4 \AA$ resolution (Bai et al. 2015) but includes all atoms of the residues and further adds loops to the structure where several of the mutations are located (Fig. 1). To investigate whether protein stability is a factor in PSEN1-variant associated FAD, mutant stabilities relative to wild-type presenilin, $\Delta \Delta \mathrm{G}(\mathrm{kcal} / \mathrm{mol})$, were calculated using the software I-Mutant 2.0 (Capriotti et al. 2005). This method is based on a support vector machine that was trained on experimental data derived from the extensive Protherm database (Gromiha 2007). We calculated the change in stability using both the homology model and the specific vector machine parameterized for protein sequence. This approach enabled an account of the role of protein stability changes associated with PSEN1 mutations causing FAD. A value of $\Delta \Delta \mathrm{G}=0$ means that the presenilin mutant has the same thermodynamic stability as the wild type protein, whereas a negative value means that it is less stable by that amount, measured in $\mathrm{kcal} / \mathrm{mol}$.

To study the possible molecular mode of action of PSEN1 mutations, changes in fundamental properties caused by the changed amino acids (such as hydrophobicity, polarity, helix propensity and empirical propensity to be buried) were computed for all PSEN1 mutants. The empirical values of these properties for each of the 20 amino acids were taken from previously standardized property scales (Grantham 1974; Kyte and Doolittle 1982; Rose et al. 1985; Roseman 1988). Because of known differences in hydrophobicity scales that could affect interpretation, we employed two commonly used but distinct scales by Roseman et al. (Roseman 1988) and Kyte and Doolittle (K\&D) (Kyte and Doolittle 1982) derived by different approaches. The numeric scales are arbitrary (e.g. derived from a weighted average of transfer free energies and the fractions of side chains buried in proteins) but the signs and magnitudes enable property correlation (Kyte and Doolittle 1982). A large positive value means that the amino acid is hydrophobic. Scales derived by Grantham et al.(Grantham 1974) and Rose et al.(Rose et al. 1985) were used to compute changes in polarity and propensity to be buried, respectively. The change in each property upon mutation was calculated as the value of the property for the specific mutant minus the value of the same property for the wildtype protein. Thus, for example, increased hydrophobicity in the mutant relative to the wild type corresponds to a positive value of the change in hydrophobicity associated with mutation.

\section{Statistical procedures}

The clinical Systematic risk modifiers, both genetic and nongenetic, strongly affect clinical age of onset, as evident from the standard deviation of onset age within single families carrying the same PSEN1 mutation typically being 2-6 years, even with up to 25 affected individuals (Poorkaj et al. 1998). Such within-family standard deviations will be a lower bound to that from a similar amount of patients from several families, where risk modifiers will be more heterogeneous. Therefore, observations based on one or

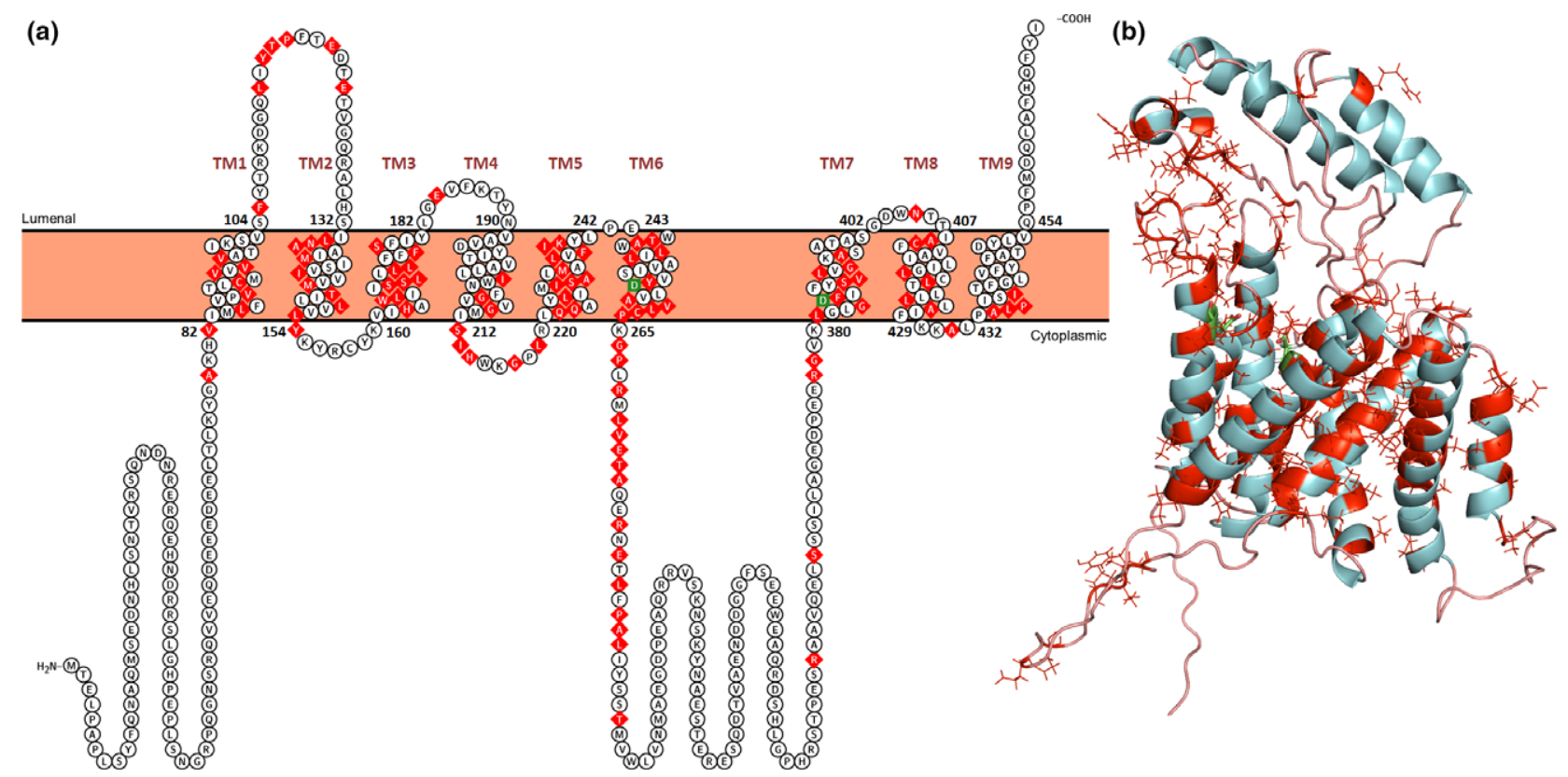

Fig. 1 (a) Topology of the transmembrane, cytoplasmic and luminal/ extracellular regions of presenilin 1 (Uniprot numbering). FAD mutations are shown in red and catalytic aspartates D257 and D385 in green. The plot was made with the PROTTER program (Omasits et al. 2014). (b) Homology model of presenilin 1 with FAD mutants mapped onto it (red lines); the catalytic aspartates are shown as green sticks. 
even two families are insignificant. The standard deviation decreases with the number $N$ of families for which data are reported. From linear regression analysis including all data where $N=1$ or 2 , no statistically significant correlations were identified, explaining why some previous work did not identify correlations between amyloid levels and age of onset (De et al. 1999; Murayama et al. 1999). Instead, we found that variant data observed in at least three families had noise levels that were small enough to provide meaningful regression; these final reduced subsets (data set 1 and 2 ) are thus relatively small but stringently defined by $N \geq 3$. The age of onset studied in these data sets ranges from 34 to 61 years.

Data sets 1 and 2 were analyzed by linear regression analysis to identify statistically significant linear correlations between changes in fundamental chemical properties and clinical data associated with each PSEN1 mutant. In each case, squared correlation coefficients $\left(R^{2}\right)$ and $p$-values of regression significance were reported. In order to be considered a significant result, $p<0.05$ was required for any such correlation, implying that the observed relationship would be $<5 \%$ likely to have arisen randomly (i.e. from a random scattering of data points). However, as seen, several of these newly identified relationships are substantially more significant than this $95 \%$ confidence level. This procedure was consistently carried out for all the chemical properties that were included in this study, as described above.

\section{Results}

Structural mapping and changes in chemical properties of PSEN1 mutants

Table S1 shows the compilation of all 183 pathogenic point missense mutations in the PSEN1 gene and our computed associated changes in chemical properties of the mutant protein. However mutations occur throughout the PSEN1 sequence, the majority of them are localized within the transmembrane regions, with TM2, TM3, TM5, TM6 and TM7 being the particularly affected regions, as seen from a mapping of mutated sites onto a membrane-topology scheme in Fig. 1a (Hardy and Crook 2001; Ogura et al. 2006; Wolfe 2013). The mapping of mutations onto the detailed homology model is shown in Fig. 1b. Clearly, many of these sites are far from the two catalytic aspartates that cleave protein substrates such as Notch and C-terminal APP fragments.

Analysis of the composition of amino acids among all pathogenic mutations reveals that the majority of the residues $(\sim 65 \%)$ that undergo mutation lose hydrophobicity. In particular, highly hydrophobic wild-type leucines are mutated into less hydrophobic residues in more than 40 (one-fifth of all) cases (Figure S1).

For each mutation, we computed hydrophobicity, the empirical propensity of the amino acid to be buried (a property that correlates with hydrophobicity), polarity (which partly correlates inversely with hydrophobicity), helix propensity and protein stability $(\Delta \Delta \mathrm{G})$ using both sequenceand structure-dependent estimates by I-Mutant 2.0 (Capriotti et al. 2005), and we assigned the solvent accessibility and secondary structure to each site. These results are shown in Table S1 of the Supporting Information.

Age of onset correlates with $A \boldsymbol{\beta}_{42} / A \boldsymbol{\beta}_{40}$ ratio

The $A \beta$ levels measured in cell models expressing mutant presenilins have been determined in many cases; these data can be directly related to the clinical age of symptom onset for specific PSEN1 variants where both types of data are available. Such correlations were previously attempted without success (De et al. 1999; Murayama et al. 1999) and with success (Duering et al. 2005; Kumar-Singh et al. 2006). A main reason for this difference is the heterogeneity in data because of risk modifiers, in particular for mild phenotypes that weaken such genotype-phenotype correlations (Kepp 2015).

As explained in the Methods, we defined two data sets for which average age of onset was available for $N \geq 3$ together with the $A \beta_{42} / A \beta_{40}$ and $A \beta_{42} / A \beta_{\text {total }}$ ratios identified in cell assays (data set 1 and 2, respectively). We then performed a linear regression analysis of these data. To be considered significant, any findings were then required to be observed in both these two independent data sets of the same approximate size. The two data sets are shown in Tables S2 and $\mathrm{S} 3$.

The regression plots are shown in Fig. 2. Significant correlations at the $95 \%$ confidence level were identified with both of the two independent data sets separately $\left(A \beta_{42} / A \beta_{40}\right.$ : $R^{2}=0.34, p=0.046$, Fig. $2 \mathrm{a} ; \mathrm{A} \beta_{42} / \mathrm{A} \beta_{\text {total }}: R^{2}=0.44$, $p=0.009$, Fig. $2 \mathrm{~b}$ ), strongly supporting a relation between pathogenicity and the ratio of $A \beta_{42}$ to $A \beta_{40}$. The significance is comparable to that which was observed previously (Duering et al. 2005; Kumar-Singh et al. 2006) and confirms that increased relative $A \beta_{42}$ levels correlate with age of symptom onset of PSEN1 variants for these two independent data sets.

\section{Chemical determinants of $A \boldsymbol{\beta}_{42} / A \boldsymbol{\beta}_{40}$ ratio}

After having confirmed that clinical age of symptom onset does indeed correlate significantly with the $A \beta_{42} / A \beta_{40}$ ratio of the PSEN1 mutants, we wanted to understand whether fundamental chemical or structural properties of the mutants could explain these correlations. To this end, we performed linear regression analysis of the chemical properties (hydrophobicity, propensity to be buried, helix propensity, polarity and protein stability) that were hypothesized to influence the conformational integrity of presenilin and could possibly explain the positional spread of PSEN1 mutations causing FAD. Numerical data of computed chemical and structural properties for the two data sets have been compiled in Tables S4 and S5.

We observed significant correlations between $A \beta_{42} / A \beta_{40}$ (Fig. 3a and b) and $A \beta_{42} / A \beta_{\text {total }}$ (Fig. $3 c$ and d) ratios and the change in hydrophobicity caused by mutation. To account for methodological differences potentially affecting 

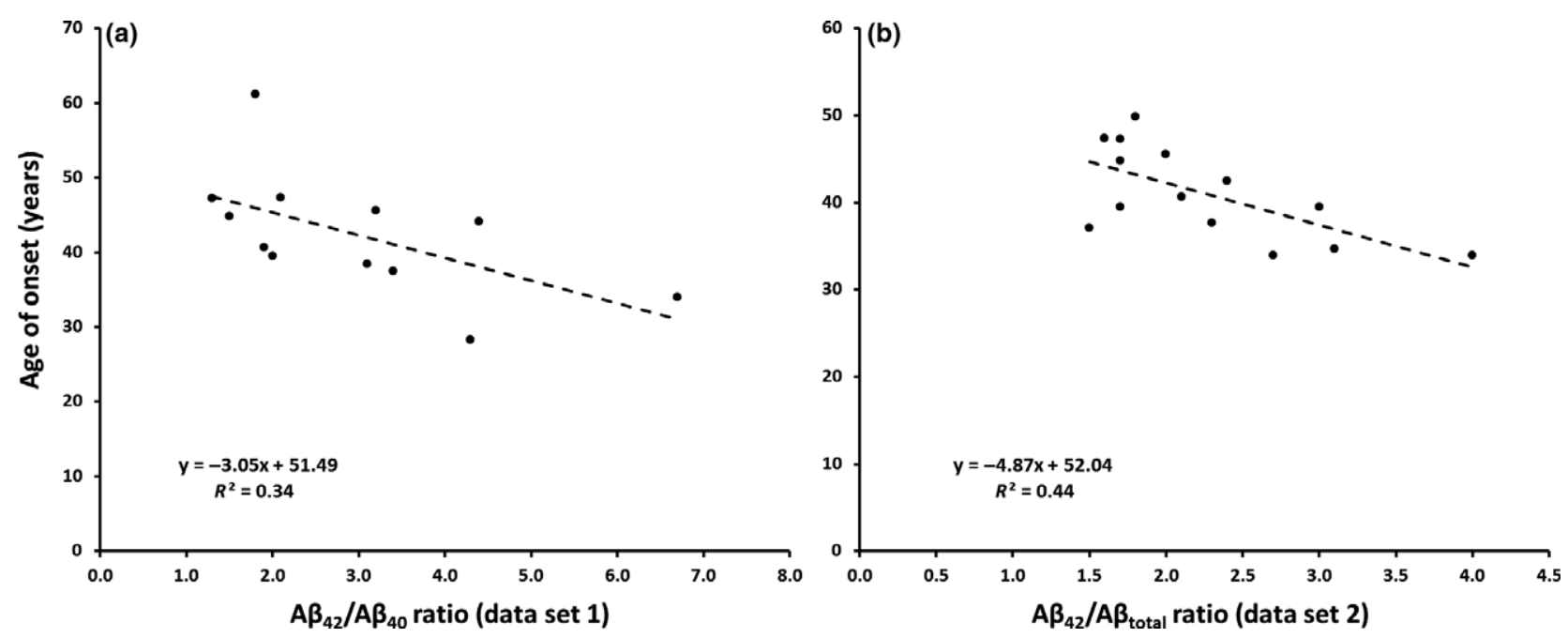

Fig. 2 Relation between age of onset of FAD and (a) the $A \beta_{42} / A \beta_{40}$ ratio for data set 1 ; (b) the $A \beta_{42} / A \beta_{\text {total }}$ ratio for data set 2 , compiled from the literature (See text for explanation).

this result, we used two different hydrophobicity scales (Roseman vs. Kyte and Doolittle). Figure $3 \mathrm{a}$ and $\mathrm{b}$ shows the correlations between $A \beta_{42} / A \beta_{40}$ ratios and the change in hydrophobicity computed using the Roseman scale $\left(R^{2}=0.51, p=0.008\right)$ and the scale by Kyte and Doolittle $\left(R^{2}=0.53, p=0.006\right)$, respectively. Figures $3 \mathrm{c}$ and $3 \mathrm{~d}$ show similar correlations using instead data set 2 with the reported $\mathrm{A} \beta_{42} / \mathrm{A} \beta_{\text {total }}$ ratios versus hydrophobicity changes according to Roseman $\left(R^{2}=0.41, p=0.012\right)$ and Kyte and Doolittle $\left(R^{2}=0.41, p=0.013\right)$. The significant correlations show that the experimentally observed increase in relative $A \beta_{42}$ levels is largely explained by decreased hydrophobicity associated with a given PSEN1 mutation. Similarly, the empirical propensity to be buried showed notable correlations for data set 2 (Figure S2). This property correlates with hydrophobicity of the amino acid and further strengthens the conclusion that this feature is important.

To further validate this fundamental relationship between chemical properties and PSEN1 phenotypes, we computed the change in polarity associated with mutation and correlated this change against experimental $A \beta_{42} / A \beta_{40}$ and $A \beta_{42} /$ $\mathrm{A} \beta_{\text {total }}$. Figures $4 \mathrm{a}$ and $\mathrm{c}$ show the linear regression plots: significant correlations were again observed for both $\mathrm{A} \beta_{42} /$ $\mathrm{A} \beta_{40}\left(R^{2}=0.47, p=0.014\right)$ and $\mathrm{A} \beta_{42} / \mathrm{A} \beta_{\text {total }}\left(R^{2}=0.59\right.$, $p=0.001$ ), with increase in polarity largely explaining the increase in relative (not absolute) $A \beta_{42}$ levels. Polarity is expected to be inversely related to hydrophobicity, so the three independently used descriptors confirm the same robust, fundamental property-phenotype relationship.

A single amino acid change in protein can interfere with solubility, stability, structure, function and substrate interactions (Steward et al. 2003). Protein misfolding and decrease in thermodynamic stability are common consequences of pathogenic missense mutations (Bross et al. 1999; Gregersen et al. 2006); impaired protein structure is a common contributor to disease (Yue et al. 2005; Chiti and Dobson 2006). To examine the effect of PSEN1 mutations on the stability of the protein, we compared the stability changes (in $\mathrm{kcal} / \mathrm{mol}$ ) computed using I-mutant 2.0 with the two sets of $\mathrm{A} \beta_{42}$ ratios. Figures $4 \mathrm{~b}$ and $\mathrm{d}$ show the regression plots with $\mathrm{A} \beta_{42} / \mathrm{A} \beta_{40} \quad\left(R^{2}=0.39, \quad p=0.028\right) \quad$ and $\mathrm{A} \beta_{42} / \mathrm{A} \beta_{\text {total }}$ $\left(R^{2}=0.63, p=0.0007\right)$ ratios both being significantly determined by stability loss associated with the mutations.

Chemical determinants of age of symptom onset

With experimental $A \beta_{42}$ levels significantly correlating with age of onset and chemical properties, it is stimulating to study any direct relationships between chemical properties and the clinical age of onset. Thus, we compared the change in properties to the clinical age of disease onset. Figures $5 \mathrm{a}$ and $\mathrm{c}$ show the regression plots for the change in polarity versus patient age of onset based on data set $1\left(R^{2}=0.49\right.$, $p=0.011)$ and data set $2\left(R^{2}=0.40, p=0.015\right)$. From this analysis, a decrease in polarity correlates with increased age of onset. To our knowledge, this is the first relationship between age of onset of PSEN1 variant carriers and fundamental chemical properties of the involved mutants. Propensity to be buried and hydrophobicity correlated less significantly but with the expected direction (Figures S2 and S3).

Correspondingly, Fig. 5b $\left(R^{2}=0.75, p=0.0002\right)$ and Fig. 5d $\left(R^{2}=0.44, p=0.009\right)$ show that the change in stability caused by mutation (in $\mathrm{kcal} / \mathrm{mol}$ ) correlates strongly with the age of onset of variant carriers, which are the most significant correlations identified in this work. The correlations to stability, polarity and hydrophobicity changes support each other and together suggest that mutations that weaken the structural integrity and membrane packing of the protein cause disease. The squared correlation coefficients 

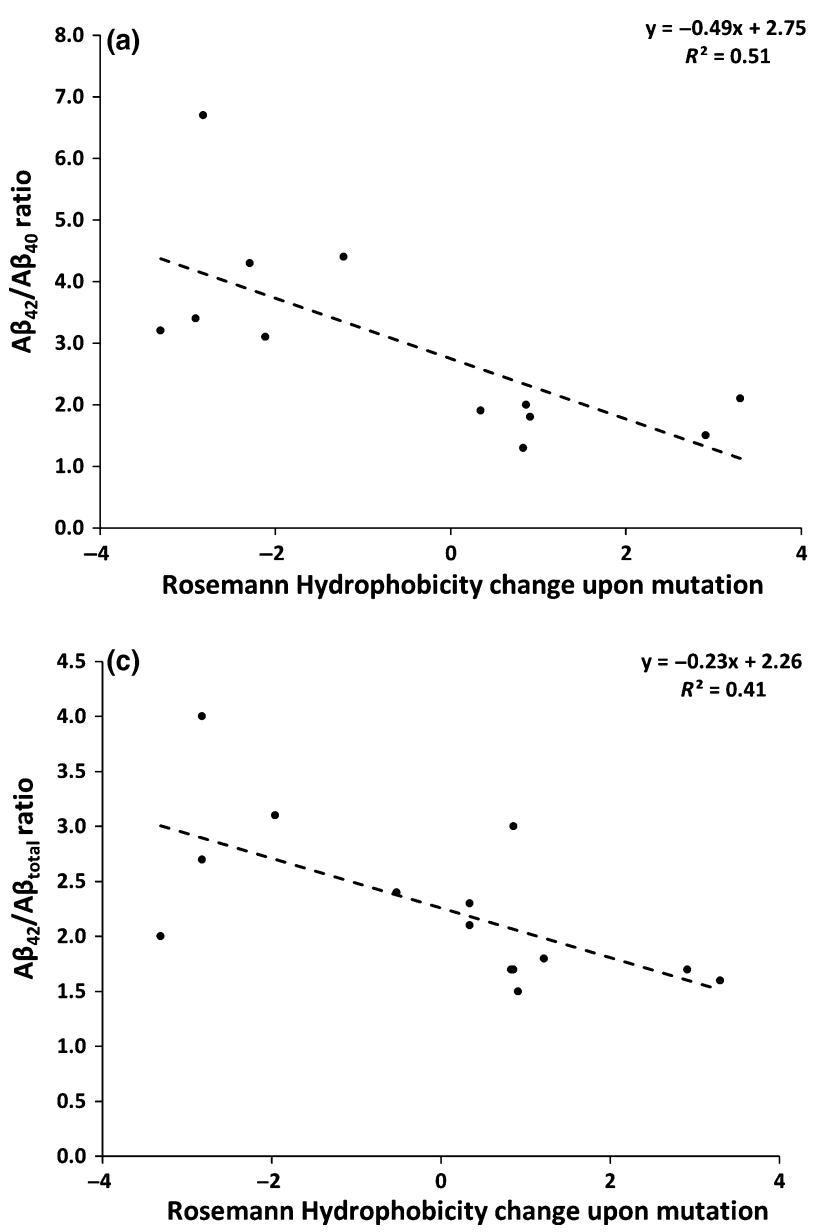

Fig. 3 Correlation between $A \beta_{42} A \beta_{40}(A, B)$ or $A \beta_{42} / A \beta_{\text {total }}$ (c and d) ratio and hydrophobicity change induced by PSEN1 mutations: (a)

and $p$-values for all the studied properties from data set 1 and 2 are shown in Tables S6 and S7, respectively.

\section{Discussion}

It has been suggested from the analysis of cleavage products that PSEN1 mutants experience reduced cleavage precision, leading to increased long-short ratios (Fukumori et al. 2010), consistent with the suggested trimming mechanism of $\gamma$ secretase (Wolfe 2012; Fernandez et al. 2014). Two explanations may be involved: (i) the catalytic turnover and/or position of substrate cleavage is very sensitive to long-range dynamics within the membrane-bound presenilin, i.e. cleavage proficiency and precision are impaired even upon marginal changes by mutation; or (ii) the general association of the protein with the membrane and substrate is sensitive to subtle variations in the protein. The first mechanism relates to $k_{\text {cat }} / K_{\mathrm{m}}$, whereas the second relates to proper formation of the mature protein complex via proper protein-membrane association.
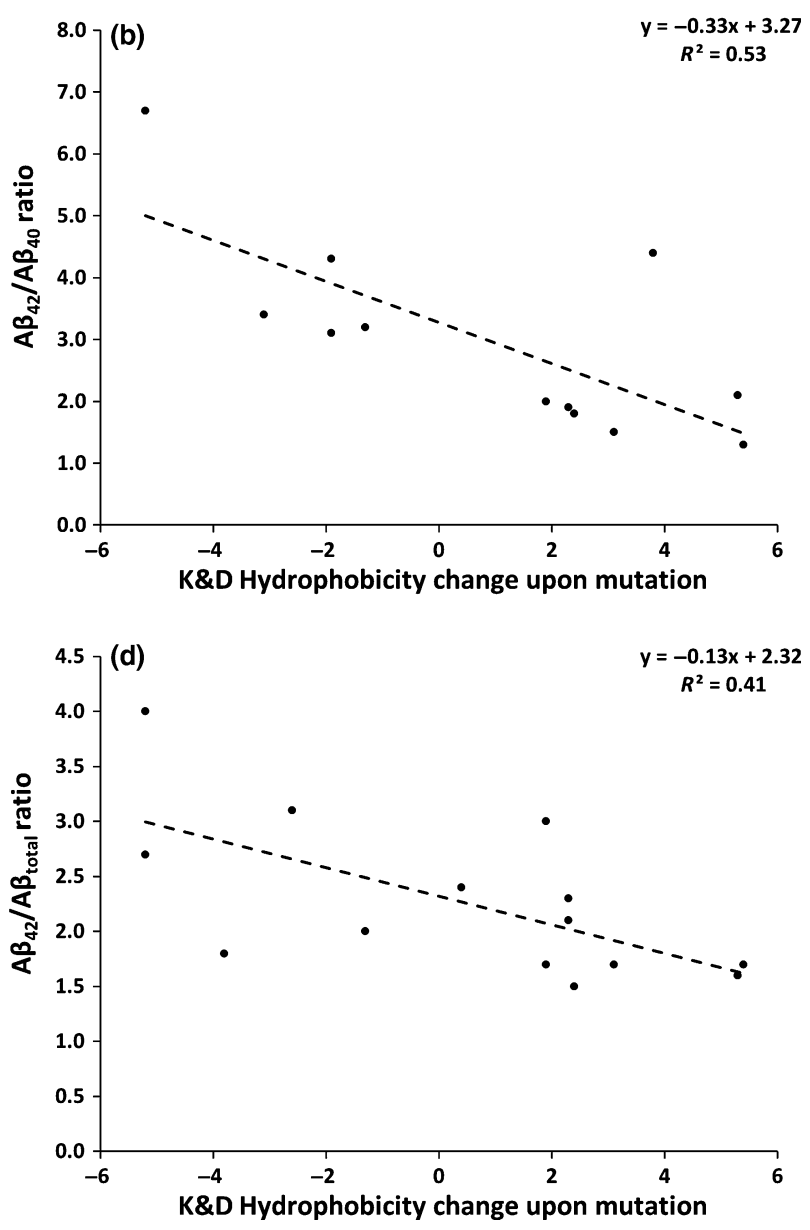

Roseman scale for data set 1; (b) K\&D scale for data set 1; (c) Roseman scale for data set 2; (d) K\&D scale for data set 2 .

The correlations in Fig. 2 show that while the relative amount (not absolute amount) of $\mathrm{A} \beta_{42}$ relates to disease, it does not mean that this relative amount causes AD (correlation does not imply causation). However, the hypothesis that relative $A \beta_{42}$ levels cause disease is consistent with and not rejected by this analysis. Still, such a hypothesis would need to explain how the increased relative amount of $A \beta_{42}$ would cause AD. Two amyloid-centric possibilities seem apparent: (i) The long-short ratio, rather than the total $A \beta$ levels, is important in seeding pathogenic oligomers, e.g. because of a competition mechanism where a certain local fraction of $A \beta_{42}$ produces pathogenic oligomers whereas a smaller local fraction does not. We refer to this mechanism as the competitive seeding mechanism (a gain of toxic $\mathrm{A} \beta_{42}$ mechanism); (ii) the higher long-short ratio changes a chemical balance relating to normal function of $A \beta$ (a loss of $\mathrm{A} \beta$ function mechanism). Third, the presenilin loss-offunction hypothesis (Shen and Kelleher 2007) would be consistent with this correlation even without the correlation being causative, if loss of presenilin function causes AD and 

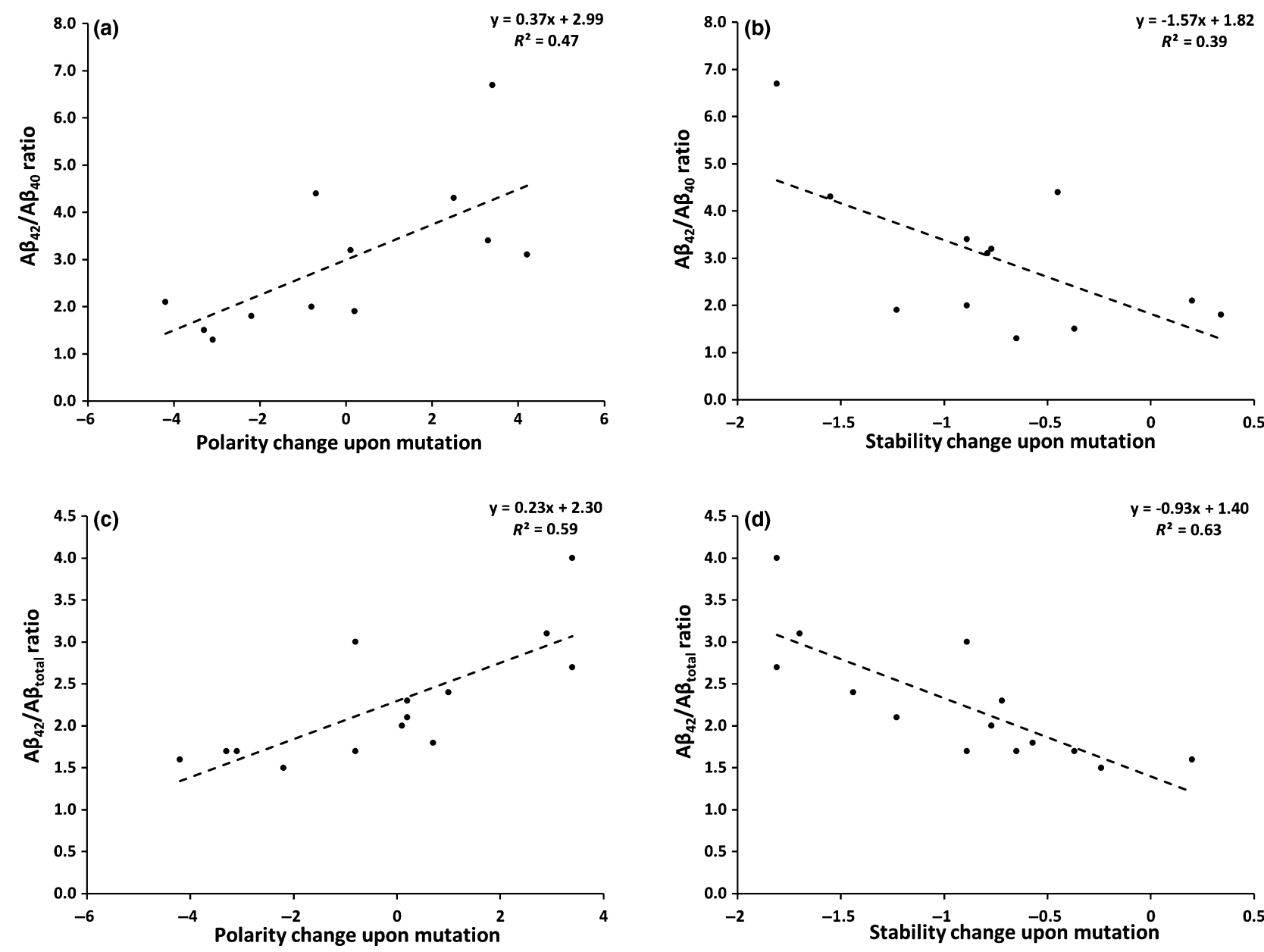

Fig. 4 (a) Correlation between $A \beta_{42} / A \beta_{40}$ from data set 1 and polarity change of PSEN1 mutations; (b) same for stability change of mutations;

the increased long-short ratio (and resulting amyloid aggregation) is a side-consequence of reduced presenilin function. Indeed, there are many more mutations in PSEN1 than APP that cause FAD and they spread throughout the sequence, whereas those in $A P P$ concentrate near the processing region (Shen and Kelleher 2007); furthermore, PSEN1 mutations are clinically more severe, in terms of early onset and rate of disease progression than APP mutations (Ryman et al. 2014).

The statistically significant correlations of our analysis presented in Fig. 3 show that the experimentally observed increase in relative $A \beta_{42}$ levels is largely explained by decreased hydrophobicity associated with a given PSEN1 mutation; this new result has immediate implications for understanding the pathogenicity of PSEN1 mutants at the molecular level.

As most of the PSEN1 mutations are associated with clinical data from only one family, and some have no age of

(c) Correlation between $A \beta_{42} / A \beta_{\text {total }}$ from data set 2 and polarity change; (d) same for stability change.

onset reported, it is of interest to see if general chemical properties are characteristic of the presenilin mutant proteins relative to the wild type presenilin. To this end, we analyzed the distribution of the chemical properties for the entire data set of PSEN1 variants (Table S1). Figures 6a and b show the distribution of the change in polarity and stability $(\Delta \Delta \mathrm{G})$, respectively. The identified chemical properties defining the pathogenicity of presenilin mutants are generally affected in the same direction when all 183 mutations are considered together. This additional analysis strengthens our suggestion that the mutations destabilize the protein, typically by increasing the polarity of the amino acid. This has two consequences: (i) the unfolded protein state becomes more favorable by additional polar residues in the presenilin chain (in contrast hydrophobic changes would render the unfolded protein less stable); and (ii) the folded mature presenilin protein will pack less well with the membrane. 

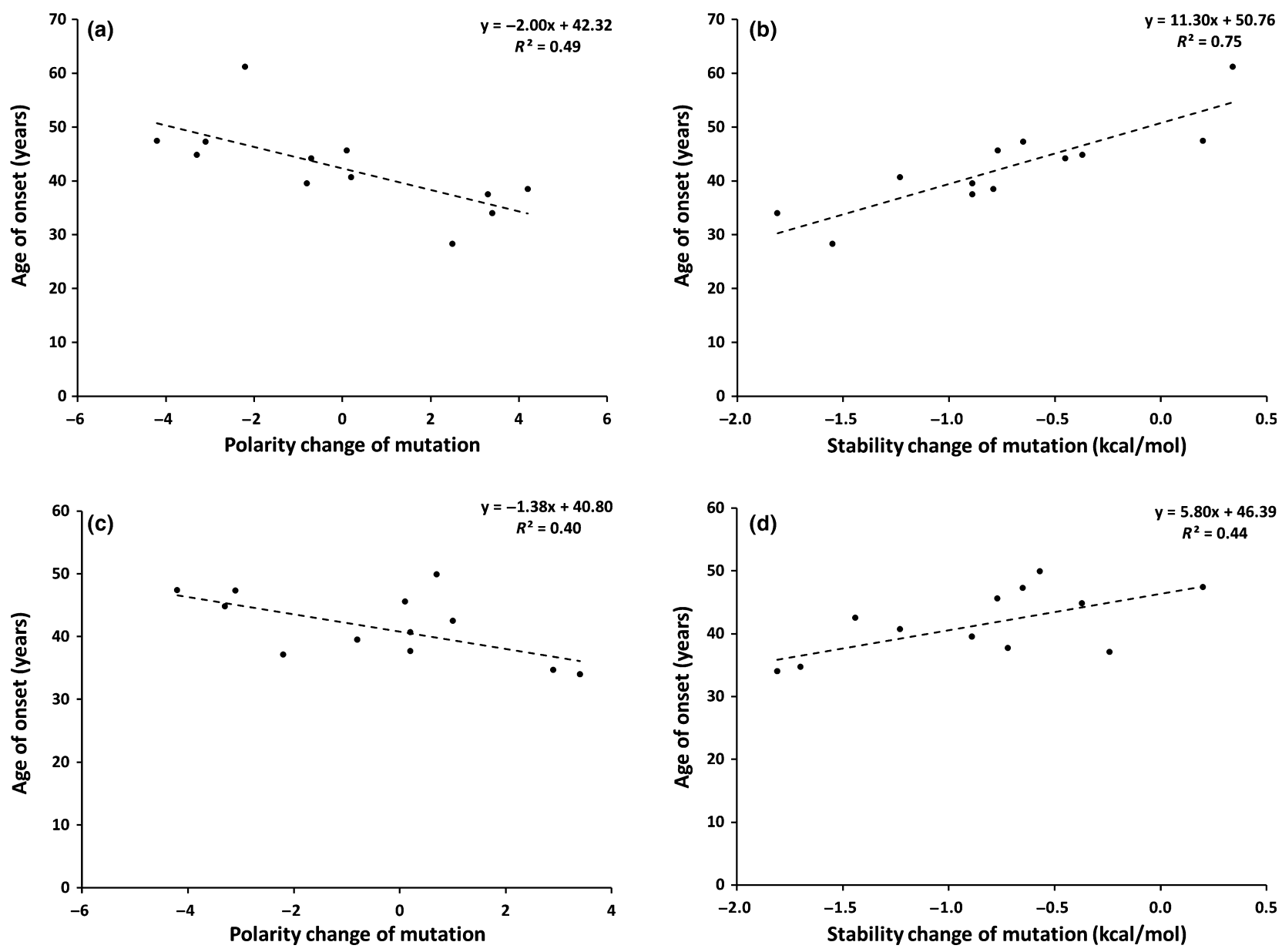

Fig. 5 Chemical drivers of PSEN1 variant pathogenicity. Age of disease onset of PSEN1 variant carriers (years) versus (a) polarity

change, data set 1 ; (b) stability change, data set 1 ; (c) polarity change, data set 2; (d) stability change, data set 2 .
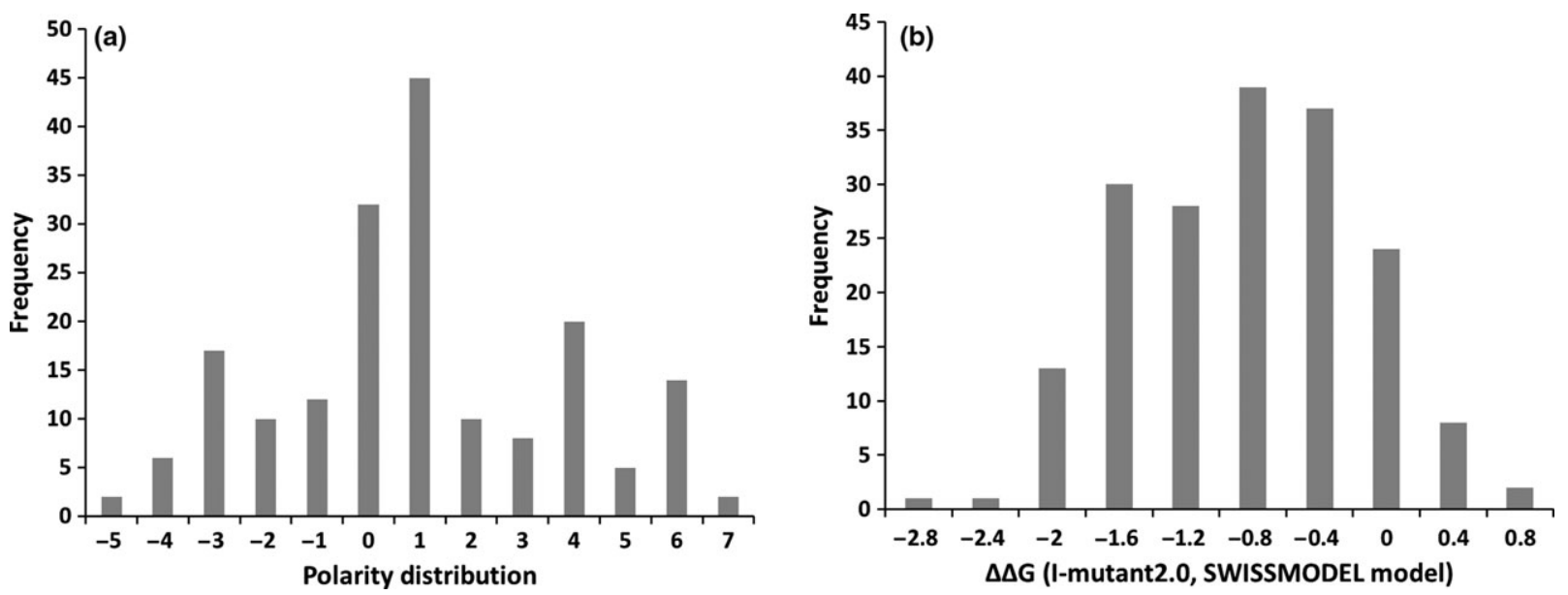

Fig. 6 Distribution of computationally estimated changes in chemical properties for 183 reported PSEN1 mutations (see text for details). (a) Estimated change in polarity; (b) Change in thermodynamic stability

caused by mutation $(\mathrm{kcal} / \mathrm{mol})$ computed using I-Mutant 2.0. and the homology model of Figure 1. 


\section{Conclusions}

In this combined meta-analysis and computational chemistry work, we studied the change in fundamental chemical properties of amino acids upon mutation of PSEN1 and compared these changes to available clinical and biochemical experimental data for PSEN1 variants known to cause Alzheimer's disease. This is the first study of its kind investigating the chemical property changes and their relation to the pathogenicity of PSEN1 mutations to establish surprising, statistically significant relations between the age of symptom onset, as a measure of the severity of pathogenesis of a genetic variant, and the hydrophobicity, polarity and stability of the corresponding mutant. Other measures of severity, such as specific histopathological phenotypes of disease characteristics of neurodegeneration, might be relevant but these are less available in the literature and thus not currently subject to analysis.

The studied properties complement each other and establish a hypothesis on the mechanism of PSEN1 mutations: the general tendency towards increased polarity or decreased hydrophobicity most likely destabilizes the membraneembedded presenilin protein. This is consistent with computed stability changes upon PSEN1 mutation. If membrane packing is impaired, the association of presenilin with other constituents of $\gamma$-secretase is likely to be reduced. Partial dissociation from the membrane and loss of compactness will potentially impair docking of APP and thus the catalytic turnover and precision of intramembrane stepwise $\varepsilon$ - and $\gamma$ cleavage (Qi-Takahara et al. 2005; Takami et al. 2009), suggesting a molecular mechanism for increased ratio of long versus short $A \beta$ peptides and the reduced overall catalytic proficiency of presenilin mutants. Our findings are in agreement with recent experiments suggesting that hydrophobic membrane thickness is a critical parameter for cleavage precision and total activity (Winkler et al. 2012). Our suggested pathogenic membrane-dissociation mechanism further explains why mutations distributed across the PSEN1 sequence can cause $\mathrm{AD}$, since overall stability and membrane association is a global property of the protein, in contrast to direct catalytic proficiency governed by a few residues close to the catalytic aspartates. This mechanism suggests new strategies for treating $\mathrm{AD}$ aiming towards specifically recovering the membrane integrity and stable, packed, membrane-bound state of presenilin.

\section{Acknowledgments and conflict of interest disclosure}

The Danish Council for Independent Research $\mid$ Natural sciences (FNU) (grant ID: DFF - 1323-00110B) and the Danish Center for Scientific Computing (grant ID: 2012-02-23) are acknowledged for supporting this work. The authors declare no conflict of interest with regard to this work.
All experiments were conducted in compliance with the ARRIVE guidelines.

\section{Supporting information}

Additional supporting information may be found in the online version of this article at the publisher's web-site:

Figure S1. Shows the composition of amino acids in wild type presenilin 1 and mutants.

Figure S2. Shows the regression analysis for the propensity of amino acids to be buried.

Figure S3. Shows the regression analysis for change in hydrophobicity versus patient age of onset.

Table S1. Shows the compiled data set of PSEN1 mutants along with the computed properties.

Tables S2 and S3. Show the pooled biochemical and clinical data (data set 1 and 2).

Tables S4 and S5. Show the computed chemical properties for data set 1 and 2 .

Tables S6 and S7. Show the squared correlation coefficients and $p$-values for the regression analysis of all studied genotypeproperty-phenotype relationships.

\section{References}

Arispe N., Rojas E. and Pollard H. B. (1993) Alzheimer disease amyloid beta protein forms calcium channels in bilayer membranes: blockade by tromethamine and aluminum. Proc. Natl Acad. Sci. USA 90, 567-571.

Arnold K., Bordoli L., Kopp J. and Schwede T. (2006) The SWISSMODEL workspace: a web-based environment for protein structure homology modelling. Bioinformatics 22, 195-201.

Bai X., Yan C., Yang G., Lu P., Sun L., Zhou R., Scheres S. H. W. and Shi Y. (2015) An atomic structure of human $\gamma$-secretase. Nature 525, 212-218.

Biasini M., Bienert S., Waterhouse A. et al. (2014) SWISS-MODEL: Modelling protein tertiary and quaternary structure using evolutionary information. Nucleic Acids Res. 42, 252-258.

Borchelt D. R., Thinakaran G., Eckman C. B. et al. (1996) Familial Alzheimer's disease-linked presenilin 1 variants elevate Abeta1-42/ 1-40 ratio in vitro and in vivo. Neuron 17, 1005-1013.

Bross P., Corydon T. J., Andresen B. S., Jørgensen M. M., Bolund L. and Gregersen N. (1999) Protein misfolding and degradation in genetic diseases. Hum. Mutat. 14, 186-198.

Bush A. I. (2013) The metal theory of Alzheimer's Disease. Rev. Lit. Arts Am. 33, 277-281.

Cacquevel M., Aeschbach L., Houacine J. and Fraering P. C. (2012) Alzheimer's disease-linked mutations in presenilin-1 result in a drastic loss of activity in purified $\gamma$-secretase complexes. PLoS ONE 7, e35133.

Campion D., Dumanchin C., Hannequin D. et al. (1999) Early-onset autosomal dominant Alzheimer disease: prevalence, genetic heterogeneity, and mutation spectrum. Am. J. Hum. Genet. 65, 664-670.

Capriotti E., Fariselli P. and Casadio R. (2005) I-Mutant2.0: predicting stability changes upon mutation from the protein sequence or structure. Nucleic Acids Res. 33, W306-W310.

Carvalho C., Correia S. C., Santos R. X., Cardoso S., Moreira P. I., Clark T. A., Zhu X., Smith M. A. and Perry G. (2009) Role of mitochondrial-mediated signaling pathways in Alzheimer disease and hypoxia. J. Bioenerg. Biomembr. 41, 433-440. 
Chávez-Gutiérrez L., Bammens L., Benilova I. et al. (2012) The mechanism of $\gamma$-Secretase dysfunction in familial Alzheimer disease. EMBO J. 31, 2261-2274.

Chiti F. and Dobson C. M. (2006) Protein misfolding, functional amyloid, and human disease. Annu. Rev. Biochem. 75, 333-366.

Corona C., Pensalfini A., Frazzini V. and Sensi S. L. (2011) New therapeutic targets in Alzheimer's disease: brain deregulation of calcium and zinc. Cell Death Dis. 2, e176.

De Strooper B. (2007) Loss-of-function presenilin mutations in Alzheimer disease. Talking Point on the role of presenilin mutations in Alzheimer disease. EMBO Rep. 8, 141-146.

De Strooper B. (2014) Lessons from a Failed $\gamma$-Secretase Alzheimer Trial. Cell 159, 721-726.

De Strooper B., Saftig P., Craessaerts K., Vanderstichele H., Guhde G., Annaert W., Von Figura K. and Van Leuven F. (1998) Deficiency of presenilin-1 inhibits the normal cleavage of amyloid precursor protein. Nature 391, 387-390.

De Jonghe C., Cras P., Vanderstichele H., Cruts M., Vanderhoeven I., Smouts I., Vanmechelen E., Martin J. J., Hendriks L. and Van Broeckhoven C. (1999) Evidence that Abeta42 plasma levels in presenilin-1 mutation carriers do not allow for prediction of their clinical phenotype. Neurobiol. Dis. 6, 280-287.

Demuro A., Parker I. and Stutzmann G. E. (2010) Calcium signaling and amyloid toxicity in Alzheimer disease. J. Biol. Chem. 285, $12463-$ 12468.

DeToma A. S., Salamekh S., Ramamoorthy A. and Lim M. H. (2012) Misfolded proteins in Alzheimer's disease and type II diabetes. Chem. Soc. Rev. 41, 608 .

Doody R. S., Raman R., Farlow M. et al. (2013) A phase 3 trial of semagacestat for treatment of Alzheimer's disease. N. Engl. J. Med. 369, 341-350.

Duering M., Grimm M. O. W., Grimm H. S., Schroder J. and Hartmann T. (2005) Mean age of onset in familial Alzheimer's disease is determined by amyloid beta 42. Neurobiol. Aging 26, 785-788.

Duff K., Eckman C., Zehr C. et al. (1996) Increased amyloid-beta42(43) in brains of mice expressing mutant presenilin 1. Nature 383, 710713.

Fernandez M. A., Klutkowski J. A., Freret T. and Wolfe M. S. (2014) Alzheimer Presenilin-1 mutations dramatically reduce trimming of long amyloid $\beta$-peptides $(\mathrm{A} \beta$ ) by $\gamma$-secretase to increase 42 -to-40residue A $\beta$. J. Biol. Chem. 289, 31043-31052.

Fukumori A., Fluhrer R., Steiner H. and Haass C. (2010) Three-amino acid spacing of presenilin endoproteolysis suggests a general stepwise cleavage of gamma-secretase-mediated intramembrane proteolysis. J. Neurosci. 30, 7853-7862.

Goate A., Chartier-Harlin M. C., Mullan M., Brown J., Crawford F., Fidani L., Giuffra L., Haynes A., Irving N. and James L. (1991) Segregation of a missense mutation in the amyloid precursor protein gene with familial Alzheimer's disease. Nature 349, 704-706.

Goedert M. and Spillantini M. G. (2006) A century of Alzheimer's Disease. Science 314, 777-781.

Grantham R. (1974) Amino acid difference formula to help explain protein evolution. Science 185, 862-864.

Gregersen N., Bross P., Vang S. and Christensen J. H. (2006) Protein misfolding and human disease. Annu. Rev. Genomics Hum. Genet. 7, 103-124.

Gromiha M. M. (2007) Prediction of protein stability upon point mutations. Biochem. Soc. Trans. 35, 1569-1573.

Haass C. and Selkoe D. J. (1993) Cellular processing of beta-amyloid precursor protein and the genesis of amyloid beta-peptide. Cell 75, 1039-1042.

Haass C., Kaether C., Thinakaran G. and Sisodia S. (2012) Trafficking and proteolytic processing of APP. Cold Spring Harb. Perspect Med. 2, a006270.
Hardy J. and Crook R. (2001) Presenilin mutations line up along transmembrane alpha-helices. Neurosci. Lett. 306, 203-205.

Hardy J. and Selkoe D. J. (2002) The amyloid hypothesis of Alzheimer's disease: progress and problems on the road to therapeutics. Science 297, 353-356.

Hefti F., Goure W. F., Jerecic J., Iverson K. S., Walicke P. A. and Krafft G. A. (2013) The case for soluble Ab oligomers as a drug target in Alzheimer's disease. Trends Pharmacol. Sci. 34, 261-266.

Hollingworth P., Harold D., Jones L., Owen M. J. and Williams J. (2011) Alzheimer's disease genetics: current knowledge and future challenges. Int. J. Geriatr. Psychiatry 26, 793-802.

Jomova K., Vondrakova D., Lawson M. and Valko M. (2010) Metals, oxidative stress and neurodegenerative disorders. Mol. Cell. Biochem. 345, 91-104.

Karran E. and Hardy J. (2014) A critique of the drug discovery and phase 3 clinical programs targeting the amyloid hypothesis for Alzheimer disease. Ann. Neurol., 185-205.

Karran E., Mercken M. and De Strooper B. (2011) The amyloid cascade hypothesis for Alzheimer's disease?: an appraisal for the development of therapeutics. Nat. Rev. Drug Discov. 10, 698-712.

Kepp K. P. (2012) Bioinorganic chemistry of Alzheimer's disease. Chem. Rev. 112, 5193-5239.

Kepp K. P. (2015) Genotype-Property Patient-Phenotype Relations Suggest that Proteome Exhaustion Can Cause Amyotrophic Lateral Sclerosis. PLOS ONE 10, e0118649.

Kimberly W. T., LaVoie M. J., Ostaszewski B. L., Ye W., Wolfe M. S. and Selkoe D. J. (2003) Gamma-secretase is a membrane protein complex comprised of presenilin, nicastrin, Aph-1, and Pen-2. Proc. Natl Acad. Sci. USA 100, 6382-6387.

Kumar-Singh S., Theuns J., Broeck B., Van Pirici D. and Vennekens K. (2006) Mean age-of-onset of familial alzheimer disease caused by presenilin mutations correlates with both increased $A \beta 42$ and decreased A $\beta 40$. Hum. Mutat. 27, 686-695.

Kuo I. Y., Hu J., Ha Y. and Ehrlich B. E. (2015) Presenilin-like GxGD membrane proteases have dual roles as proteolytic enzymes and Ion channels. J. Biol. Chem. 290, 6419-6427.

Kyte J. and Doolittle R. F. (1982) A simple method for displaying the hydropathic character of a protein. J. Mol. Biol. 157, 105-132.

Lemere C. A., Lopera F., Kosik K. S. et al. (1996) The E280A presenilin 1 Alzheimer mutation produces increased A beta 42 deposition and severe cerebellar pathology. Nat. Med. 2, 1146-1150.

Levy-Lahad E., Wasco W., Poorkaj P. et al. (1995) Candidate gene for the chromosome 1 familial Alzheimer's disease locus. Science 269, 973-977.

Lu P., Bai X., Ma D. et al. (2014) Three-dimensional structure of human $\gamma$-secretase. Nature 512, 166-170.

Morris J. K., Honea R. A., Vidoni E. D., Swerdlow R. H. and Burns J. M. (2014) Is Alzheimer's disease a systemic disease? Biochim. Biophys. Acta - Mol. Basis Dis. 1842, 1340-1349.

Murayama O., Tomita T., Nihonmatsu N., Murayama M., Sun X., Honda T., Iwatsubo T. and Takashima A. (1999) Enhancement of amyloid beta 42 secretion by 28 different presenilin 1 mutations of familial Alzheimer's disease. Neurosci. Lett. 265, 61-63.

Ogura T., Mio K., Hayashi I. et al. (2006) Three-dimensional structure of the $\gamma$-secretase complex. Biochem. Biophys. Res. Commun. 343, 525-534.

Omasits U., Ahrens C. H., Müller S. and Wollscheid B. (2014) Protter: interactive protein feature visualization and integration with experimental proteomic data. Bioinformatics 30, 884-886.

Poorkaj P., Sharma V., Anderson L., Nemens E., Alonso M. E., Orr H., White J., Heston L., Bird T. D. and Schellenberg G. D. (1998) Missense mutations in the chromosome 14 familial Alzheimer's disease presenilin 1 gene. Hum. Mutat. 11, 216-221. 
Qi-Takahara Y., Morishima-Kawashima M., Tanimura Y. et al. (2005) Longer forms of amyloid beta protein: implications for the mechanism of intramembrane cleavage by gamma-secretase. $J$. Neurosci. 25, 436-445.

Rauk A. (2009) The chemistry of Alzheimer's disease. Chem. Soc. Rev. 38, 2698-2715.

Rogaev E. I., Sherrington R., Rogaeva E. A., Levesque G., Ikeda M., Liang Y., Chi H., Lin C., Holman K. and Tsuda T. (1995) Familial Alzheimer's disease in kindreds with missense mutations in a gene on chromosome 1 related to the Alzheimer's disease type 3 gene. Nature 376, 775-778.

Rose G. D., Geselowitz A. R., Lesser G. J., Lee R. H. and Zehfus M. H. (1985) Hydrophobicity of amino acid residues in globular proteins. Science 229, 834-838.

Roseman M. A. (1988) Hydrophilicity of polar amino acid side-chains is markedly reduced by flanking peptide bonds. J. Mol. Biol. 200 , 513-522.

Rosenblum W. I. (2014) Why Alzheimer trials fail: removing soluble oligomeric beta amyloid is essential, inconsistent, and difficult. Neurobiol. Aging 35, 969-974.

Ryman D. C., Acosta-Baena N., Aisen P. S. et al. (2014) Symptom onset in autosomal dominant Alzheimer disease: A systematic review and meta-analysis. Neurology 83, 253-260.

Sali A. and Blundell T. L. (1993) Comparative protein modelling by satisfaction of spatial restraints. J. Mol. Biol. 234, 779-815.

Saura C. A., Choi S.-Y., Beglopoulos V. et al. (2004) Loss of presenilin function causes impairments of memory and synaptic plasticity followed by age-dependent neurodegeneration. Neuron 42, 23-36.

Shen J. and Kelleher R. J. (2007) The presenilin hypothesis of Alzheimer's disease: evidence for a loss-of-function pathogenic mechanism. Proc. Natl Acad. Sci. USA 104, 403-409.

Sherrington R., Rogaev E. I., Liang Y. et al. (1995) Cloning of a gene bearing missense mutations in early-onset familial Alzheimer's disease. Nature 375, 754-760.

Small D. H., Gasperini R., Vincent A. J., Hung A. C. and Foa L. (2009) The role of $\mathrm{A} \beta$-induced calcium dysregulation in the pathogenesis of Alzheimer's disease. J. Alzheimer's Dis. 16, 225-233.

Steward R. E., MacArthur M. W., Laskowski R. A. and Thornton J. M. (2003) Molecular basis of inherited diseases: a structural perspective. Trends Genet. 19, 505-513.
Sun L., Zhao L., Yang G. et al. (2015) Structural basis of human $\gamma$ secretase assembly. Proc. Natl Acad. Sci. USA 112, 6003-6008.

Supnet C. and Bezprozvanny I. (2011) Presenilins function in ER calcium leak and Alzheimer's disease pathogenesis. Cell Calcium 50, 303-309.

Takami M., Nagashima Y., Sano Y., Ishihara S., MorishimaKawashima M., Funamoto S. and Ihara Y. (2009) gammaSecretase: successive tripeptide and tetrapeptide release from the transmembrane domain of beta-carboxyl terminal fragment. $J$. Neurosci. 29, 13042-13052.

Teich A. F. and Arancio O. (2012) Is the Amyloid Hypothesis of Alzheimer's disease therapeutically relevant? Biochem. J. 446, $165-177$.

Tiwari M. K. and Kepp K. P. (2015) $\beta$-Amyloid pathogenesis: chemical properties versus cellular levels. Alzheimer's Dement. 9, 1-11.

Wilkins H. M., Carl S. M., Greenlief A. C. S., Festoff B. W. and Swerdlow R. H. (2014) Bioenergetic dysfunction and inflammation in Alzheimer's disease: a possible connection. Front. Aging Neurosci. 6, 311.

Winkler E., Kamp F., Scheuring J., Ebke A., Fukumori A. and Steiner H. (2012) Generation of Alzheimer disease-associated amyloid $\beta 42 /$ 43 peptide by $\gamma$-secretase can be inhibited directly by modulation of membrane thickness. J. Biol. Chem. 287, 21326-21334.

Wolfe M. S. (2012) Processive proteolysis by $\gamma$-secretase and the mechanism of Alzheimer's disease. Biol. Chem. 393, 899-905.

Wolfe M. S. (2013) Toward the structure of presenilin/ $\gamma$-secretase and presenilin homologs. Biochim. Biophys. Acta - Biomembr. 1828, 2886-2897.

Woodruff G., Young J. E., Martinez F. J., Buen F., Gore A., Kinaga J., Li Z., Yuan S. H., Zhang K. and Goldstein L. S. B. (2013) The Presenilin-1 $\delta$ E9 mutation results in reduced $\gamma$-secretase activity, but not total loss of PS1 function, in isogenic human stem cells. Cell Rep. 5, 974-985.

Yue P., Li Z. and Moult J. (2005) Loss of protein structure stability as a major causative factor in monogenic disease. J. Mol. Biol. 353, 459-473. 